\title{
Determinantes biológicos e ambientais no desenvolvimento neuropsicomotor em uma amostra de crianças de Canoas/RS
}

\author{
Environmental and biological determinants of neuropsychomotor \\ development in a sample of children in Canoas/RS
}

Elsa M aria Luz Pilz ${ }^{1}$

Lígia Braun Schermann ${ }^{2}$
Artigo baseado na dissertação de mestrado da primeira autora, apresentada no Programa dePós-Graduação em Saúde Coletiva, Universidade Luterana do Brasil, Canoas, RS.

${ }^{1}$ Programa de PósGraduação em Saúde Coletiva, Universidade Luterana do Brasil. Rua Miguel Tostes Prédio 14/ sala 22. Bairro São Luís. 92420-280 Canoas RS epilz@terra.com.br 2 Universidade Luterana do Brasil.
Abstract The object of this study is to determine the prevalence of potential delaysin neuropsychomotor development and their possible association with, on one hand, environmental and biological factors, and maternal competence on the other, in a sample of children up to six years old living in Canoas, in Rio Grande do Sul state. A questionnaire was submitted to mothers including questions on social, economic and reproduction factors; child's conditions at birth; child's pathologies; family structure; child care and elements on maternal competence. The potential for neuropsychomotor development delay was assessed by the Denver II Test. Forty clusters were visited in Canoas, a city in Rio Grande do Sul state, in accordance with the cluster probabilistic sampling process. From 197 children assessed by this analytical cross-section study, there was a $27 \%(n=53)$ prevalence of potential delay in neuropsychomotor development. The multivariate analysis showed that factors associated with potential development delays were: low income (or $=9,3)$; mothers with less than 18-month intervals between pregnancies (or $=3,9)$; and lack of support from child's father (or $=7,0)$. These results support the importance of implementing income generating programs, health education, and family planning in order to prevent child development delays.

Key words Environment factors, Biological factors, $\mathrm{M}$ aternal competence, Child development
Resumo Este estudo verificou a prevalência de suspeita de atraso no desenvolvimento neuropsicomotor e possíveis associações a fatores ambientais e biológicos, bem como à competência materna, em uma amostra de crianças de até 6 anos residentes no município de Canoas/RS. Às mães foi aplicado um questionário sobre fatores socioeconômicos e reprodutivos, condições da criança ao nascer, patologia da criança, estrutura familiar, atenção à criança e componentes da competência materna. Avaliou-se a suspeita de atraso no desenvolvimento neuropsicomotor via teste de Denver II. V isitaram-se quarenta clusterspelo processo de amostragem probabilística por cluster na cidade de Canoas/RS. 0 delineamento do estudo foi analíti co transversal. Segundo os resultados, há prevalência de suspeita de atraso no desenvolvimento neuropsicomotor de 27\% (n=53) das 197 crianças avaliadas. A análise multivariada mostrou os seguintes fatores associados à suspeita de atraso no desenvolvimento: baixa renda familiar (or $=9,3)$, gestação materna com intervalo interpartal inferior a 18 meses (or $=3,9)$ e mães sem 0 apoio dos pais da criança $($ or $=7,0)$. Os resultados afirmam a importância de programas de geração de renda, educação em saúde e planejamento familiar para a prevenção de atraso no desenvolvimento infantil.

Palavras-chave Fatores ambientais, Fatores biológicos, Competência materna, Desenvolvimento infantil 
Introdução

As crianças que vivem em países em desenvolvimento estão expostas a múltiplos riscos, entre os quais o de apresentarem uma alta prevalência de doenças, o de nascerem de gestações desfavoráveis e/ou incompletas e o de viverem em condições socioeconômicas adversas. Tal cadeia deeventos negativos faz com que essas crianças tenham maior chance de apresentar atrasos em seu potencial de crescimento e desenvolvimento ${ }^{1,2}$. Estudos demonstram que as formas pelas quais se manifesta a dificuldade quanto ao desenvolvimento infantil podem estar relacionadas a fatores biológicos, genéticos, psicológicos e ambientais, geralmente envolven do interações complexas entre eles ${ }^{3}$. Por essa razão, o impacto de fatores biológicos, psicossociais (individuais e familiares) e ambientais no desenvolvimento infantil tem sido objeto de inúmeros estudos nas últimas décadas 4,5 .

Historicamente, os estudos sobre desenvolvimento têm colocado as características biológicas da população infantil como determinante principal dos atrasos intelectuais da criança. Tais idéias podem ser verdadeiras para crianças gravemente comprometidas ${ }^{6,7}$, porém, não para a maioria das crianças que apresentam um atraso moderado ou leve no seu desenvolvimento ${ }^{2}$. Um único evento, exceto se resultar em dano orgânico irreversível, não épreditivo de desfecho de de senvolvimento. A identificação de uma criança de risco pode ocorrer pela natureza do ambiente no qual ela nasceu, ou por sua condição orgânica ou por ambos os fatores ${ }^{8}$.

Um problema biológico pode ser agravado por um ambiente não-estimulador, mas, por outro lado, pode também ser reduzido por um ambiente de apoio. Os múltiplos fatores envolvidos na determinação dos problemas de desenvolvimento e comportamento infantis são mais dependentes da quantidade do que da natureza dos fatores de risco, visto que diferentes fatores de risco produzem resultados semelhantes?.

A pobreza, por exemplo, é considerada um tipo de ameaça constante, que aumenta a vulne rabilidade da criança, pois pode causar subnutrição, privação social e desvantagem educacional ${ }^{10}$. Pesquisadores ressaltam que crescer na pobreza consiste uma ameaça ao bem- estar da criança e numa limitação de suas oportunidades de desenvolvimento. A miséria econômica é um fator de risco que, em determinadas situações, não vem desacompanhado; ela permeia a relação conjugal, contribuindo para o aumento da incidência de conflitos entre os pais e produzindo um efeito direto no relacionamento desses com a criança. Desta forma, ocasiona o que alguns autores denominam como miséria afetiva ${ }^{11}$.

Na maioria dos estudos conduzidos nas áreas do desenvolvimento infantil ederelações sociais, a interação entremãee criança tem sido considerada um recorte importante para o estudo da organização comportamental e competências do bebê, adquiridas no contexto desta relação. Desde a concepção, mães e bebês participam de um sistema muito complexo de relações, o qual emerge, se organiza e se modifica através do curso da evolução e de eventos culturais interpostos ao desenvolvimento subseqüente de ambos. $A$ qualidade da interação inicial é considerada um importante fator mediador entre os eventos perinatais e 0 seu posterior desenvolvimento, particularmente no que se refere à comunicação, social ização e cogniçãa ${ }^{12}$.

Diversas teorias psicológicasincluem em seus pressupostos quea natureza das experiênciascom os cuidadores durante a infância exerce influência no desenvolvimento cognitivo, emocional e social da criança ${ }^{13,14,15}$. U ma vez que em nossa sociedade, em geral, éa mãea principal cuidadora do(a) filho(a), uma mãe competente pode ser uma fonte rica em estímulos, podendo desencadear respostas adequadas da criança ${ }^{13,16}$. No presente estudo, a competência materna é considerada um construto composto por quatro componentes (rede de apoio, satisfação marital, saúde materna e característica da criança), que interligados, tornam-seum exemplo claro da complexa interação de fatores ambientais e biológicos na determinação do desenvolvimento infantil.

Segundo Tarkka ${ }^{17}$, em um estudo sobre os determinantes da competência materna em mães primíparas, utilizou-se um questionário estruturado que foi aplicado em 248 mães. Realizada análise multivariada, a autora elaborou um modelo com determinantes para o estudo da competência materna, dos quais os mais importantes foram a saúde mental da mãe, o sentimento de sucesso nos cuidados com a criança e o temperamento da criança. A autora concluiu que a competência materna é fortemente influenciada pelo contexto familiar e cultural da mãe, pelas características da criança e da mãe, bem como pelo suporte social recebido pela mãe.

Outro estudo examinou os determinantes da competência materna em 58 mães adolescentes. As mães respondiam um questionário que contemplava perguntas sobre auto-estima, 
eventos de vida, suportesocial, bem- estar ecompetência materna, bem como eram filmadas em atividades junto a seus filhos. 0 estudo mostrou que o suporte do pai da criança era negativamente associado com estresse da mãe e positivamente associado com auto-estima e eficácia da mãe. Àquelas com maior suporte do pai da criança tinham melhor auto- estima e maior sentimento de eficácia ${ }^{18}$.

Em um estudo longitudinal, em que participaram 45 mães e seus filhos de 16 a 62 meses de idade, a satisfação marital foi examinada como um determinante adicional na relação mãe-criança. 0 relacionamento marital pode prover à mãeo suporteemocional eprático queela necessita, ten do forte impacto na sensitividade da mãe com seu filho. Deste modo, havendo satisfação na relação marital, há menor tensão em casa, propiciando um ambiente onde a criança passa aincorporar o estilo interacional dos pais ${ }^{19}$. M ães querecebem mais suporte deseus companheiros têm comportamento positivo com seus filhos, influindo na qualidade das atividades que elas desempenham com a criança ${ }^{15}$.

Identificar etratar as dificuldades relacionais da criança e de seus pais tornou-se um objetivo prioritário em saúde pública e isso vem sendo confirmado pelo sucesso dos serviços destinados a esta população. Toda estratégia de prevenção deve intervir o mais cedo possível, assim que fatores de risco são identificados e, idealmente, antes mesmo do surgimento das primeiras dificuldades $^{20}$. 0 impacto positivo que os programas de intervenção precoce tem mostrado em relação ao desenvolvimento da criança e posterior desempenho escolar justificam a necessidade da identificação precoce das crianças com risco de atrasos ${ }^{21}$.

Sendo assim, o presente estudo teve por objetivo verificar a prevalência de suspeitas de atraso no desenvolvimento neuropsicomotor e possíveis associações com fatores ambientais, biológicos, bem como com competência materna em uma amostra de crianças de 0 a 6 anos de idade, residentes no município deCanoas/RS. A identificação dessesfatores possibilitará, posteriormente, elaborar intervenções adequadas e voltadas para a promoção da saúde materno-infantil no município de Canoas/RS.

\section{M aterial e método}

Trata-se de um estudo analítico observacional transversal realizado no município deCanoas-RS.
Este estudo utilizou o mesmo processo de amostragem probabilística por "cluster" de uma pesquisa patrocinada pela O rganização Mundial da Saúde, "Prevalência de Surdez e Outros Transtornos da Audição" 22. Foram sorteados aleatoriamente 40 dos 391 setores censitários do município de Canoas/RS. Em cada setor, foi sorteado um quarteirão e deste foi sorteada uma esquina. A partir desta esquina, foram visitados 26 domicílios, sistematicamente de dois em dois. Desta forma, para realizar este estudo, todas as residências com crianças de 0 a 6 anos de idade foram revisitadas. Compuseram a amostra do presenteestudo 197 crianças com idade de 0 a 6 anos e respectivas mães.

Para realização da coleta de dados, foram recrutados universitários dos cursos de medicina e psicologia, além deuma psicóloga eumafonoaudióloga. Esta equipe de entrevistadores foi treinada na administração do teste D enver II e para aplicação do questionário às mães. Foi realizado estudo piloto com 25 coletas de dados, o que permitiu reproduzir as condições nas quais o estudo seria desenvolvido efazer uma avaliação do treinamento dos entrevistadores tanto na aplicação do teste, como na entrevista com as mães. Além disso, uma amostra aleatória de $5 \%$ dos casos foi revisitada com a finalidade de verificar a qualidade e veracidade das informações e os critérios utilizados na pontuação dos itens.

O questionário foi construído para fins específicos desteestudo econtemplou questões socioeconômicas: renda familiar, escolaridade materna; reprodutivas: idade materna, intervalo interpartal, pré-natal, problemas na gestação; condições da criança ao nascer: peso ao nascer, idade gestacional, morbidade neonatal; patologia da criança: hospitalização no primeiro ano; estrutura familiar: pai mora junto, número de irmãos; aten ção à criança: freqüenta ou freqüentou creche, aleitamento materno; saúde materna: uso de medicação psiquiátrica, presença de problemas de saúde; satisfação marital: está satisfeita com o relacionamento conjugal, está satisfeita com a ajuda do marido/companheiro nos cuidados com a criança, presença de brigas conjugais; rededeapoio: apoio do pai da criança, apoio de familiares, apoio de não familiares; características da criança: como a mãe avalia o comportamento da criança quanto ao sono, choro, fome, atividade, ambientenovo, ao ser contrariada, presença de amigos.

O questionário continha, ainda, uma medida de percepção da competência materna, conforme a opinião expressa pela mãe quanto a ser 
pouco competente ou muito competente como mãe do(a) seu(ua) filho(a) em uma linha ótica dedez centímetros.

Para identificar a criança com suspeita de atraso no desenvolvimento neuropsicomotor, foi utilizado o testeDenver $\mathrm{II}^{23}$. 0 teste, delineado para ser utilizado em crianças desde o nascimento até a idade de 6 anos, consiste em 125 itens que são divididos em quatro grupos: a) pessoal-social b) motricidade fina c) linguagem d) motricidade ampla. Esses itens são registrados através da observação direta da criança e, para al guns deles, solicita-seque a mãe informese o fil ho realiza ou não determinada tarefa. Foram considerados casos indicativos de suspeita de atraso no desenvolvimento aqueles em que a criança apresentou dois ou mais itens defal ha, isto é, não realização do item quando $90 \%$ ou mais das crianças da faixa etária o realiza, independente da área em que ocorreu. A variável dependente suspeita de atraso no desenvolvimento neuropsicomotor foi tratada como variável dicotômica (D enver II, suspeito versus não suspeito).

Para a análise dos dados, foi realizada a análise bivariada por meio do teste do qui quadrado para caracterizar os grupos suspeitos e os não suspeitos de atraso no desenvolvimento. A seguir, foi realizada a análise multivariada utilizando o método de regressão logística, cuja modela- gem obedeceu um modelo hierárquico de determinação (Figura 1).

0 uso deste modelo estabelece que as diversas variáveis potencialmente associadas ao desfecho apresentam uma relação proximal ou distal com o desfecho; portanto, tais variáveis podem produzir confusão no estabelecimento de uma possível associação entre as variáveis estudadas ${ }^{24}$. 0 pacoteestatístico utilizado para a análise foi o SPSS 10.0 for W indows.

Este estudo foi aprovado pelo ComitêdeÉtica da Universidade Luterana do Brasil, eestá deacordo com as normas vigentes na Resolução $\mathrm{N}$ o 196/ 96 do Conselho Nacional de Saúde/M inistério da Saúde, e suas complementares, que regulamentam a pesquisa envolvendo seres humanos.

\section{Resultados}

Na população estudada, $27 \%(n=53)$ das crianças apresentaram teste de triagem de Denver II suspeito deatraso no desenvolvimento. A distribuição por sexo mostra que, dentre os suspeitos, $60 \%(n=32)$ são do sexo masculino e $40 \%(n=21)$ são do sexo feminino. As crianças suspeitas de atraso apresentavam em média 41 meses (desvio padrão de 18 meses) e as não suspeitas de atraso em média 54 meses (desvio padrão de 23 meses).

Figura 1

M odelo teórico de determinação de suspeita de atraso no desenvolvimento neuropsicomotor emcrianças de 0 a 6 anos de idade, segundo teste Denver II.

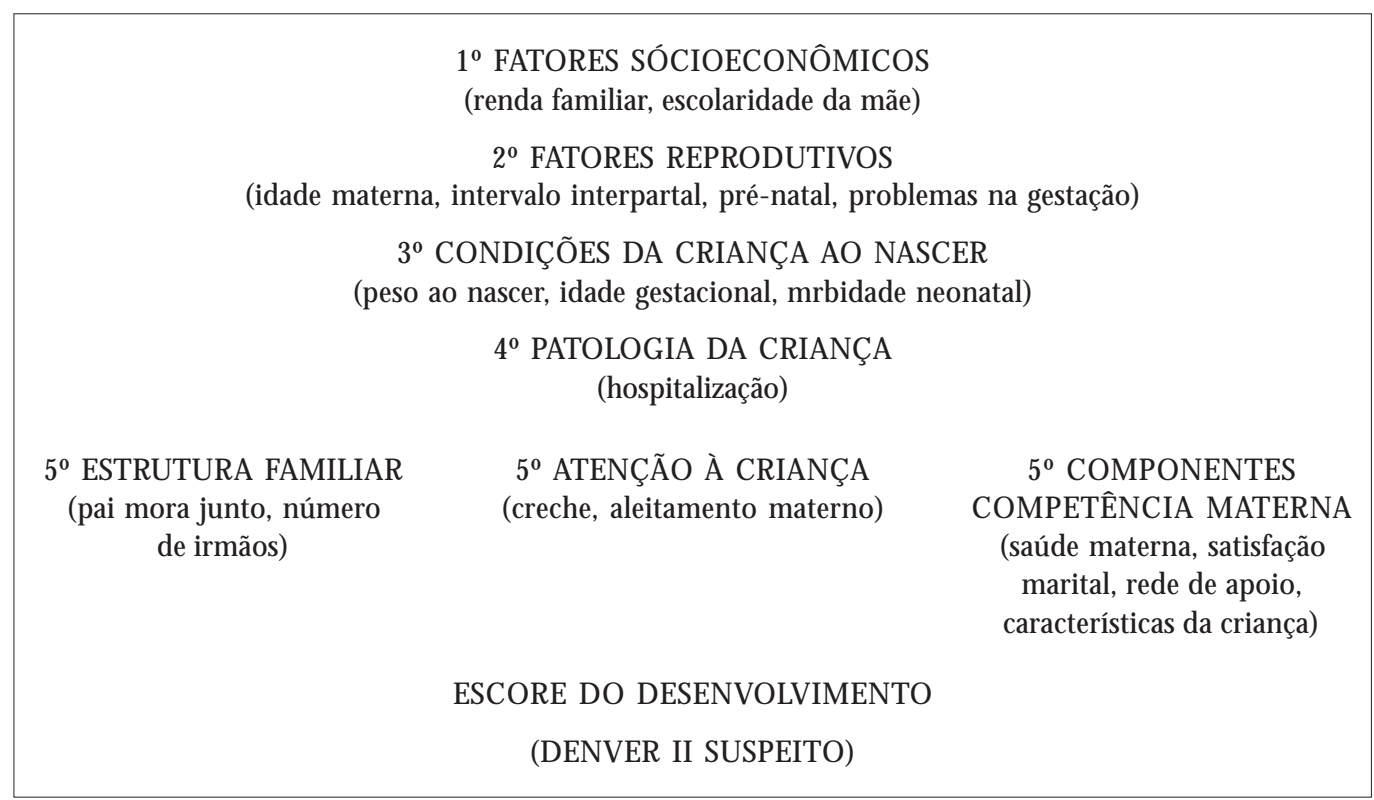


A Tabela 1 apresenta a distribuição dos dados referente à análise bivariada e o percentual de suspeita de atraso no desenvolvimento neuropsicomotor em cada estrato das variáveis estudadas. As variáveis independentes que mostraram associação significativa com suspeita de atraso no desenvolvimento neuropsicomotor na análise bivariada foram: renda familiar, crianças de famílias com renda de atéum salário mínimo mensal mostraram 15,1 vezes mais chance de apresentar suspeita deatraso no desenvolvimento neuropsicomotor quando comparadas com crianças de famílias com renda maior do que três salários mínimos; escolaridade da mãe, crianças de mães com até três anos de estudo mostraram 6,0 vezes mais chance de apresentar suspeita de atraso no desenvolvimento quando comparadas com crianças de mães com nove ou mais anos de estudo; número deirmãos, crianças com três ou mais irmãos mostraram 6,4 vezes mais chance de apresentar suspeita de atraso no desenvolvimento quando comparadas com as que não possuem irmãos; intervalo interpartal, crianças de gestações com intervalo interpartal menor de 18 meses mostraram 6,9 vezes mais chance de apresentar suspeita de atraso no desenvolvimento; problemas maternos na gestação (diabete melito, pressão alta, infecção urinária, ameaça de aborto eanemia), crianças cujas mães apresentaram dois problemas na gestação mostraram 3,5 vezes mais chance de apresentar suspeita de atraso no desenvolvimento; hospitalização, crianças que tiveram histórico de hospitalização mostraram 7,0 vezes mais chance de apresentar suspeita de atraso no desenvolvimento.

As variáveis incluídas nos componentes da competência materna queisoladamenteapresentaram significância estatística na análise bivariadaforam: a) apoio do pai nos cuidados da criança, crianças cujos pais não apoiam as mães nos cuidados com os filhos mostraram 12,2 vezes mais chance apresentar suspeita de atraso no desenvolvimento; b) brigas conjugais, crianças cujos pais brigam mostraram 4,4 vezes maischance de apresentar suspeita de atraso no desenvolvimento; c) uso de medicação psiquiátrica, crianças de mães que fazem uso deste tipo de medicação mostraram 4,2 vezes mais chance de apresentar suspeita de atraso no desenvolvimento; d) presença de amigos, crianças com idade superior a 2 anos eque não possuem amigos mostraram 3,6 vezes mais chance de apresentar suspeita de atraso no desenvolvimento. A percepção da competência materna, avaliada por meio da opinião da mãe quanto a ser pouco competente ou muito competente como mãe do(a) seu(ua) filho(a), expressa em uma linha ótica de 10 centímetros, foi categorizada em quatro faixas: até $40 \mathrm{~mm}$, de 41 a $60 \mathrm{~mm}$, de 61 a $80 \mathrm{~mm}$ e acima de $80 \mathrm{~mm}$. A análise bivariada mostrou que, dentre as mães das crianças com suspeita de atraso no desenvolvimento, $20 \%$ expressaram opinião acima de $80 \mathrm{~mm}$ quanto a sua competência como mãe de seus filhos, enquanto que mães de crianças sem suspeita de atraso no desenvolvimento expressam esta opinião em $80 \%$.

O ajuste para possíveis variáveis de confusão foi realizado por meio de análise multivariada, segundo o modelo hierarquizado proposto. Cada nível hierárquico foi inserido isoladamentenaregressão logística para verificar as possíveis associações com a variável dependente. No modelo final, permaneceram apenas as variáveisindependentes cuja regressão logística mostrou significância estatística. 0 resultado desta análise encontra-se na Tabela 2.

Crianças de famílias com renda de até um salário mínimo mostraram uma probabilidade 9,3 vezes maior de apresentarem suspeita de atraso no desenvolvimento neuropsicomotor do que crianças de família com renda maior do que três salários mínimos. Da mesma forma, crianças inseridas em uma família cujo intervalo interpartal é de até 18 meses possuem uma probabilidade 3,9 vezes maior de apresentarem suspeita de atraso do desenvolvimento neuropsicomotor do que crianças cujas famílias apresentam intervalo interpartal maior do que 18 meses. Por fim, a probabilidade de crianças cujas mães não recebem apoio dos pais apresentarem suspeita de atraso no desenvolvimento neuropsicomotor é 7,0 vezes maior do que aquelas cujas mães possuem apoio dos pais.

\section{Discussão}

Neste estudo, a renda familiar foi o fator que demonstrou maior associação com a suspeita de atraso no desenvolvimento neuropsicomotor. Este resultado reafirma o que vem sendo preconizado por organizações internacionais, como, por exemplo, a Organização das Nações Unidas, que definiu como uma das prioridades para a agenda global do século XXI a erradicação da extrema pobreza e da fome ${ }^{25}$. Resultado semeIhante foi encontrado por $\mathrm{Halpern}$ et al. ${ }^{26}$, em estudo que avaliou 1.363 crianças aos 12 meses nascidas em Pelotas/RS, em 1993. Os autores verificaram uma prevalência de suspeita de atraso 
Tabela 1

Análise bivariada entre as variáveis independentes e o desfecho suspeita de atraso no desenvolvimento neuropsicomotor.

\begin{tabular}{|c|c|c|c|c|c|c|}
\hline Variável & $\mathrm{N}$ & $\begin{array}{c}\% \text { da } \\
\text { amostra }\end{array}$ & $\begin{array}{c}\% \text { de } \\
\text { suspeitos }\end{array}$ & $\begin{array}{l}\text { Valor } \\
\text { dep }\end{array}$ & $\begin{array}{l}\text { Odds } \\
\text { Ratio }\end{array}$ & IC $95 \%$ \\
\hline \multicolumn{7}{|l|}{ Renda familiar } \\
\hline > 3 salários mínimos & 54 & 27 & 9 & & 1,0 & $(3,01-28,55)$ \\
\hline 0 a 1 salários mínimos & 37 & 19 & 48 & 0,00 & 15,11 & $(1,23-10,61)$ \\
\hline 1,1 a 2 salários mínimos & 63 & 32 & 27 & 0,01 & 5,50 & $(1,37-13,10)$ \\
\hline 2,1 a 3 salários mínimos & 43 & 22 & 30 & 0,01 & 6,32 & \\
\hline Total & 197 & 100 & 27 & & & \\
\hline \multicolumn{7}{|l|}{ Escolaridade da mãe } \\
\hline 9 ou mais anos & 61 & 31 & 18 & & 1,0 & $(1,40-21,13)$ \\
\hline 0 a 3 anos & 11 & 6 & 54 & 0,01 & 6,02 & $(0,86-3,92)$ \\
\hline 4 a 8 anos & 125 & 63 & 29 & 0,04 & 2,47 & \\
\hline Total & 197 & 100 & 27 & & & \\
\hline \multicolumn{7}{|l|}{ Idade da mãe } \\
\hline $19-39$ & 165 & 84 & 27 & & 1,0 & $(1,58-6,34)$ \\
\hline $13-18$ & 8 & 4 & 25 & 1,00 & 0,00 & $(0,42-3,01)$ \\
\hline 40 anos ou mais & 24 & 12 & 25 & 0,81 & 0,05 & \\
\hline Total & 197 & 100 & 27 & & & \\
\hline \multicolumn{7}{|l|}{ Intervalo interpartal } \\
\hline 19 meses ou mais & 175 & 94 & 24 & & 1,0 & $(1,54-19,86)$ \\
\hline até 18 meses & 11 & 6 & 63 & 0,00 & 6,9 & \\
\hline Total & 186 & 94 & 26 & & & \\
\hline \multicolumn{7}{|l|}{ Pré-natal } \\
\hline sim & 190 & 98 & 26 & & 1,0 & $(0,01-1,17)$ \\
\hline não & 4 & 2 & 75 & 0,06 & 3,32 & \\
\hline Total & 194 & 98 & 27 & & & \\
\hline \multicolumn{7}{|l|}{ Problemas maternos } \\
\hline nenhum & 112 & 58 & 28 & & 1,0 & $(0,01-1,27)$ \\
\hline 1 problema & 59 & 29 & 24 & 0,07 & 3,07 & $(0,01-1,07)$ \\
\hline 2 problemas & 22 & 11 & 23 & 0,05 & 3,59 & $(0,00-1,16)$ \\
\hline 3 problemas & 4 & 2 & 75 & 0,06 & 3,38 & \\
\hline Total & 197 & 100 & 27 & & & \\
\hline \multicolumn{7}{|l|}{ Peso ao nascer } \\
\hline$>2500 \mathrm{gr}$ & 179 & 91 & 27 & & 1,0 & $(1,14-1,86)$ \\
\hline$\leq 2500 \mathrm{gr}$ & 18 & 9 & 16 & 0,30 & 0,60 & \\
\hline Total & 197 & 100 & 27 & & & \\
\hline \multicolumn{7}{|l|}{ Idade gestacional } \\
\hline$\geq 37$ semanas & 161 & 79 & 28 & & 1,0 & $(0,31-1,93)$ \\
\hline$<37$ semanas & 30 & 15 & 23 & 0,59 & 0,29 & \\
\hline Total & 191 & 97 & 27 & & & \\
\hline \multicolumn{7}{|l|}{ M orbidade neonatal } \\
\hline sem problemas & 174 & 88 & 28 & & 1,0 & $(0,54-2,86)$ \\
\hline 1 problema & 15 & 8 & 8 & 0,35 & 0,85 & $(0,00-1,20)$ \\
\hline 2 problemas & 4 & 2 & 25 & 0,06 & 3,33 & $(0,01-6,65)$ \\
\hline 3 problemas & 4 & 2 & 50 & 0,47 & 0,51 & \\
\hline Total & 197 & 100 & 27 & & & \\
\hline \multicolumn{7}{|l|}{ H ospitalização } \\
\hline Não & 140 & 72 & 22 & & 1,0 & $(1,26-4,90)$ \\
\hline Sim & 53 & 28 & 41 & 0,01 & 7,01 & \\
\hline Total & 197 & 100 & 27 & & & \\
\hline
\end{tabular}

continua 
Tabela 1

continuação

\begin{tabular}{|c|c|c|c|c|c|c|}
\hline Variável & $\mathrm{N}$ & $\begin{array}{c}\% \text { da } \\
\text { amostra }\end{array}$ & $\begin{array}{c}\% \text { de } \\
\text { suspeitos }\end{array}$ & $\begin{array}{l}\text { Valor } \\
\text { de } p\end{array}$ & $\begin{array}{l}\text { Odds } \\
\text { Ratio }\end{array}$ & IC $95 \%$ \\
\hline \multicolumn{7}{|l|}{ Número de irmãos } \\
\hline nenhum & 47 & 24 & 17 & & 1,0 & \\
\hline 1 a 2 & 107 & 54 & 25 & 0,47 & 3,95 & $(0,22-0,98)$ \\
\hline 3 ou mais & 43 & 22 & 42 & 0,01 & 6,40 & $(0,10-0,75)$ \\
\hline Total & 197 & 100 & 27 & & & \\
\hline \multicolumn{7}{|l|}{ Pai mora junto } \\
\hline Sim & 153 & 78 & 24 & & 1,0 & \\
\hline Não & 44 & 22 & 36 & 0,11 & 2,54 & $(0,27-1,14)$ \\
\hline Total & 197 & 100 & 27 & & & \\
\hline \multicolumn{7}{|c|}{ Frequenta ou frequentou creche } \\
\hline Sim & 47 & 24 & 17 & & 1,0 & \\
\hline Não & 150 & 76 & 30 & 0,08 & 2,97 & $(0,20-1,10)$ \\
\hline Total & 197 & 100 & 27 & & & \\
\hline \multicolumn{7}{|l|}{ Aleitamento materno } \\
\hline Sim & 173 & 88 & 26 & & 1,0 & \\
\hline Não & 23 & 12 & 30 & 0,65 & 0,20 & $(0,48-3,22)$ \\
\hline Total & 196 & 99 & 26 & & & \\
\hline \multicolumn{7}{|c|}{ M ãe recebe o apoio do pai da criança } \\
\hline Sim & 101 & 51 & 15 & & 1,0 & \\
\hline Não & 96 & 49 & 38 & 0,00 & 12,24 & $(0,15-0,58)$ \\
\hline Total & 197 & 100 & 27 & & & \\
\hline \multicolumn{7}{|l|}{ Presença de brigas conjugais } \\
\hline Não & 74 & 76 & 17 & & 1,0 & \\
\hline Sim & 23 & 24 & 39 & 0,03 & 4,41 & $(1,07-8,44)$ \\
\hline Total & 97 & 100 & 23 & & & \\
\hline \multicolumn{7}{|c|}{ Uso de medicação psiquiátrica } \\
\hline Não & 61 & 75 & 36 & & 1,0 & \\
\hline Sim & 20 & 25 & 10 & 0,04 & 4,21 & $(0,42-0,92)$ \\
\hline Total & 81 & 41 & 29 & & & \\
\hline \multicolumn{7}{|l|}{ Presença de amigos } \\
\hline Sim & 165 & 92 & 25 & & 1,0 & \\
\hline Não & 14 & 8 & 50 & 0,05 & 3,63 & $(0,11-1,03)$ \\
\hline Total & 179 & 91 & 27 & & & \\
\hline \multicolumn{7}{|c|}{$\begin{array}{l}\text { M edida da percepção da competência } \\
\text { materna }\end{array}$} \\
\hline acima de $80 \mathrm{~mm}$ & 89 & 45 & 20 & 0,00 & 11,91 & \\
\hline até $40 \mathrm{~mm}$ & 12 & 6 & 33 & 0,30 & 1,03 & $(0,53-7,28)$ \\
\hline de 41 a $60 \mathrm{~mm}$ & 51 & 26 & 19 & 0,93 & 0,00 & $(0,40-2,28)$ \\
\hline de 61 a $80 \mathrm{~mm}$ & 45 & 23 & 47 & 0,00 & 9,65 & $(1,58-7,53)$ \\
\hline Total & 197 & 100 & 27 & & & \\
\hline
\end{tabular}

no desenvolvimento neuropsicomotor de 34\% da amostra. $\mathrm{Na}$ análise multivariada, após controle das variáveis de confusão, os autores mostraram que as crianças que tinham maior risco de suspeita de atraso em seu desenvolvimento foram: as mais pobres, as que haviam nascido com mais baixo peso, as que apresentaram idade gestacional menor que 37 semanas, as que ti- nham mais de três irmãos e as que tinham recebido leite materno por menos de três meses ou não haviam sido amamentadas.

A forte associação encontrada entre a baixa renda familiar e a suspeita de atraso no desenvolvimento reforça a característica multifatorial do desenvolvimento infantil e o conceito de efeito cumulativo de risco ${ }^{3}$. Bebês de mulheres com 
dietas pobres em nutrientes têm maior probabilidade de terem baixo peso ao nascer, de sofrer comprometimento do desenvolvimento cerebral, de ser menos resistente a doenças e de terem um risco mais alto de mortalidade no primeiro ano de vida ${ }^{27}$.

Segundo M artins et al. ${ }^{28}$, em um estudo prospectivo cujo objetivo foi descrever e identificar fatores associados à qualidade do ambiente $e$ características das crianças expostas, em Pelotas/ RS, no ano de 1998, foram avaliadas 630 crianças da coorte de nascimentos de 1993. Os autores encontraram que renda familiar mensal inferior a um salário mínimo constitui um fator de risco associado à qualidade do ambiente $(O R=4,5)$. 0 estudo concluiu que a renda familiar é determinante para a qualidade de vida das famílias quanto ao acesso à saúde, educação, alimentação e habitação, entre outros.

D entre as variáveis referentes à competência materna, o apoio do pai da criança foi o componente que permaneceu no modelo final de regressão logística. Os pais, além de condições materiais, ao darem apoio emocional à mãe, por meio do amor ecompanheirismo, ajudam a manter um clima de harmonia e satisfação no qual a criança se desenvolve, promovendo um desenvolvimento saudável ${ }^{15,16}$. O suporte que o companheiro fornece para a esposa influi no afeto materno, tendo um efeito protetor, fazendo com que a mulher tenha melhor auto-estima e maior sentimento de eficácia na sua função materna ${ }^{18,19}$.

Em um estudo na cidade de Brasília, ${ }^{29}$ investigaram a rede social de apoio durante as transi- ções familiares decorrentes do nascimento de fiIhos. Participaram desse estudo quinze pais de classe social baixa esuas respectivas esposas/companheiras. A principal alteração narededeapoio, segundo as mães, foi o aumento do apoio psicológico recebido; para os pais, foi o aumento da ajuda financeira e material. 0 apoio recebido do marido/companheiro foi considerado por todas as mães como o mais importante.

D entre as variáveis reprodutivas investigadas no presente estudo, o intervalo interpartal permaneceu no modelo final de regressão logística. O intervalo interpartal curto está relacionado com aumento da mortalidade neonatal e infanti ${ }^{30}$. A atenção dedicada às crianças, cujas mães tiveram muitos filhos, com pequeno intervalo inerpartal, é dificultada ${ }^{31}$.

A variável problemas na gestação não semanteve no modelo final de regressão logística do presente estudo, embora estudos mostrem que mulheres que apresentam problemas durante a gestação, como pressão alta, diabete melito, anemia e infecções do trato renal, têm maior taxa de mortalidade neonatal em seus bebês ${ }^{32}$. Da mesma forma, as variáveis escolaridade materna, hospitalização da criança e número de irmãos, embora tenham mostrado associação significativa na análise bivariada, não permaneceram no modelo final de regressão logística. As variáveis da competência materna que isoladamente merecem destaque, embora não tenham permanecido no modelo final de regressão logística, são: brigas conjugais, uso de medicação psiquiátrica e presença de amigos.

Tabela 2

Análise multivariada: regressão logística ajustada para o modelo final. Desfecho considerado suspeita de atraso no desenvolvimento.

\begin{tabular}{lcccc}
\hline Variável & OR Bruto & OR Ajustado & Valor dep & IC (95\%) \\
\hline Renda familiar & & & & \\
> 3 SM & 1,0 & 1,0 & & \\
0 a 1 & 15,1 & 9,3 & 0,00 & $(1,94-20,64)$ \\
1,1 a 2 & 5,5 & 3,3 & 0,06 & $(0,92-8,96)$ \\
2,1 a 3 & 6,3 & 5,9 & 0,01 & $(1,34-14,19)$ \\
Intervalo interpartal & 1,0 & 1,0 & & \\
$\quad$ 19 meses ou mais & 6,9 & 3,9 & 0,04 & $(1,02-24,08)$ \\
Até 18 meses & 1,0 & 1,0 & & \\
Apoio do pai & 12,2 & 7,0 & 0,00 & $(0,17-0,76)$ \\
Sim & & & & \\
Não & & & & \\
\hline
\end{tabular}


A percepção da competência materna avaliada por meio da opinião da mãe quanto a ser pouco competente ou muito competente mostrou significância estatística na análise bivariada. A penas 20\% das mães das crianças suspeitas de atraso julgam ser competentes como mães em uma medida acima de $80 \mathrm{~mm}$ em comparação a $80 \%$ das mães das crianças sem suspeita de atraso. Um estudo com 48 mães clinicamente deprimidas e 38 mães não deprimidas igualmente mostrou que o senso de auto-eficácia materna serve de mediador na relação entre competência materna, avaliada através do senso de auto-eficácia e outras variáveis psicossociais e pode ter um papel crucial na determinação do comportamento parental e risco psicossocial infantili3.

Em relação ao tamanho da amostra, este estudo apresentou limitações devido às perdas ocorridas no número de crianças avaliadas (um total de $29 \%$ ). As perdas ocorreram devido à impossibilidade de entrevistar os sujeitos da pesquisa em lugares em que não foi possível o acesso dos pesquisadores, por serem descritos como zonas de invasão, e também devido à mudança de endereço para outras cidades e estados. Essa dificuldade em conseguir a amostra completa pode ter levado a um erro do tipo II, queéa não determinação de uma associação que na realidadeexiste.
As famílias não avaliadas tiveram, porém, seu perfil descrito deacordo com os dados da pesquisa "Prevalência de Surdez e outros transtornos da audição" ${ }^{23}$. Essas famílias não apresentaram diferença estatisticamentesignificativa em relação à amostra deste estudo, concluindo-se, portanto, que os resultados obtidos podem ser generalizados para a amostra prevista inicialmente.

0 presente estudo mostrou que crianças que vivem em situação de pobreza, que nasceram em famílias com pequeno intervalo interpartal ecujas mães não possuem apoio dos pais nos cuidados com os filhos estão expostas a fatores de risco e vulnerabilidade que podem trazer efeitos negativos para o seu desenvolvimento físico, cognitivo epsicossocial. A saúdeéresultantedecondições dignas de moradia, acesso à cultura, ao esporte, ao lazer e à educação, além do cuidado com aspectos biológicos. Podemos concluir que o estudo do desenvolvimento infantil passa pela compreensão das múltiplas conexões na qual a criança está inserida e são inúmeros os fatores associados ao atraso no desenvolvimento. Para a implementação de programas de promoção da saúde, é preciso considerar a complexidade e a dinâmica das políticas públicas que estão relacionadas às oportunidades e às condições socioeconômicas e culturais no desenvolvimento das crianças.

\section{Colaboradores}

EM L Pilz trabalhou na pesquisa de campo, banco de dados, revisão bibliográfica e redação do texto; LB Schermann trabalhou na concepção teórica e redação final do texto. 


\section{Referências}

1. Victora CG, Barros FC, Vaughan JP. Epidemiologia da desigualdade. São Paulo: Ed. Hucitec; 1989.

2. Escalona S K. Babies at double hazard: early development of infants at biologic and social risk. Pediatrics 1982; (70):670-6.

3. Halpern R, Figueiras ACM . Influências ambientais na saúde mental da criança. Jornal de Pediatria 2004; 80(2):104-110.

4. Ruter M. Pathways from childhood to adult life. J Child Psychol Psychiatry 1989; 30(1): 23-51.

5. Garbarino J. The human ecology of early risk. In: M eisels SJ, editors. Handbook of early childhood intervention. M elbourne: Cambridge University Press; 1990. p.78-96.

6. Whitaker $\mathrm{AH}$, et al. Neonatal cranieal ultrasound abnormalities in low birth weigth infants: relation to cognitive outcomes at six years of age. Pediatrics 1996; 98(4):719-29.

7. Koller $\mathrm{H}$, Lowsonk RSA, Wallace I, McCarton C Patterns of cognitive development in very low birth wergth children during the first six years of life. Pediatrics 1997; (99):383-9.

8. Horowitz FD. Exploring developmental theories: toward a structural/behavioral model of development. New Jersey: Erlbaum; 1987.

9. Rae-Grant N, Thomas BH, Offord DR ,Boyle MH Risk, protective factors, and the prevalence of behavioral and emotional didorders in children and adolescents. J Am Acad Child Adolesc Psychiatry 1989; 28(2):262-8.

10. Zimmerman MA, Arunkumar R. Resiliency research:implications for schoolsand policy. Soc Policy Rep 1994; 8(1):1-18.

11. Hutz CS, Koller SH, Bandeira DR. Resiliência e vulnerabilidade em crianças em situação de risco. Coletâneas da AN PEPP 1996; 1(12):79-86.

12. Zamberlan MAT. Interação mãe-criança: enfoques teóricos e implicações decorrentes de estudos empíricos. Estudos de Psicologia ( $\mathrm{Natal}$ ) 2002; 7(2):399-406.

13. Bowlby J. U ma base segura: aplicações clínicas da teoria do apego. Porto Alegre; Artes M édicas; 1989.

14. Brazelton TB. 0 desenvolvimento do apego: uma família em formação. Porto Alegre: Artes M édicas; 1988.

15. Klaus MH, Kennell JH. Vínculo: construindo as bases para um apego seguro e para a independência. Porto Alegre: Artes M édicas; 2000.

16. Stern D. Constelação da maternidade. Porto Alegre: Artes M édicas; 1997

17. Tarkka TM. Predictors of maternal competence by first-time mothers when the child is 8 months old. J Adv Nurs 2003; 41(3): 233-240.

18. Shapiro RJ, M angelsdorf CS. The determinants of parenting competence in adolescent mothers. Journal of Youth and Adolescent 1994; 23(6):621-649.

19. Eiden RD, Corns M K, Tetti MD. M aternal working models of attachment, marital adjustment, and parent-child relationship. Child Dev 1995; (66):15041518.
20. Wendland J. Cuidando do bebê e sua família no período perinatal: abordagens de prevenção e de intervenção precoce na U nité Enfance Vivaldi. In: Filho LC, Corrêa MEG, França OS, organizadores. N ovos olhares sobre a gestação e a criança até os 3 anos. Brasília: LGE/ Apoio Funsaúde; 2002. p. 512-535.

21. Baydar N, Reid MJ, Webster-Stratton C. The role of mental health factors and program engagement in the effectiveness of a preventive parenting program for Head Start mothers. Child Dev 2003; 74(5):1433-1453.

22. Béria J, Raymann B, Gigante L. Prevalência de surdez e outros transtornos de audição: um estudo de base populacional no Sul do Brasil. Anais do Congresso Brasileiro de Saúde Coletiva. Brasília: Abrasco; 2000.

23. Frankenburg KW, et al. Denver II: Technical manual and training manual. Denver: Denver Developmental Materials; 1990.

24. Fuchs SC, Victora CG, Fachel J. Hierarchical Model: a proposal for a model to be applied in the investigation of risk factor for severe diarrhea. Rev Saúde Pública 1996; 30(2):168-178.

25. UNESCO. A UNESCO e os objetivos de desenvolvimento do milênio. Disponível em: http://www. unesco.org.br/unesco/nomundo/odm

26. Halpern R, Giugliani ERJ, Victora CG, Barros FC, $\mathrm{H}$ orta BL. Fatores de risco para suspeita de atraso no desenvolvimento neuropsicomotor aos 12 meses de vida. Jornal de Pediatria 2000; 76(6):421-428.

27. Newcombe D. Desenvolvimento infantil: uma abordagem de M ussen. Porto Alegre: Artemed; 1999.

28. Martins M FD, Costa JSD, Saforcada ET, Cunha MDC. Qualidade do ambiente e fatores associados: um estudo em crianças de Pelotas, Rio Grande do Sul, Brasil. Cad Saúde Pública 2004; 20(3):710-718.

29. Dessen MA, Braz MP. Rede social de apoio durante transições familiares decorrentes do nascimento de fiIhos. Psicologia: Teoria e Pesquisa 2000; 16(3): 221-231.

30. Wang IY, Fraser IS. Reproductive function and contraception in the postpartum period. Obstet. Gynec.Surv. 1994; 49(1): 56-63.

31. Shimakura SE, Carvalho MS, Aerts DR, Flores R. Distribuição espacial do risco: modelagem da mortalidade infantil em Porto Alegre, RS, Brasil. Cad Saúde Pública 2001; 17(5):1251-1261.

32. Naufal J. Gestação de alto risco. In: Basseto MCA, Brock $R$, Wajnsztejn $R$, organizadores. Neonatalogia: um convite à atuação fonoaudiológica. São Paulo: Lovise; 1998. p. 35-40.

33. Tetti MD, Gelfand MD. Behavioral competence among mothers of infants in the first year: the mediational role of maternal self-efficacy. Child Dev 1991; (62):918-929.

Artigo apresentado em 25/07/2005

Aprovado em 29/08/2005

Versão final apresentada em 9/02/2006 